\title{
Neurite growth inhibitors restrict plasticity and functional recovery following corticospinal tract lesions
}

Michaela Thallmair, Gerlinde A.S. Metz, Werner J. Z'Graggen, Olivier Raineteau, Gwendolyn L. Kartje and Martin E. Schwab

Nature Neurosci. 1, 124-131 (1998).

We reported in the June 1998 issue of Nature Neuroscience that the monoclonal antibody IN-1 promotes collateral sprouting in the rat spinal cord, red nucleus and pons following a lesion to the corticospinal tract. This sprouting was accompanied by functional recovery. We also published a paper in the 15 June 1998 issue of Journal of Neuroscience (Vol. 18: 4744-4757), which described experiments that were performed in parallel in our laboratory and reached similar conclusions; specifically, whereas the Nature Neurosci. paper concentrated on spinal effects, the J. Neurosci. paper employed identical lesions and antibody treatments, and described in detail sprouting in red nucleus and pons as well as functional recovery. Although the datasets are largely unique to each paper, the experimental design and results were very similar. Moreover, some of the data presented in the two papers are also identical (Fig. $5 \mathrm{~b}$ of the Nature Neurosci. paper and Fig. 4a of the J. Neurosci. paper), while other data represent different time points from the same animals (Fig. $6 \mathrm{~b}$ of the Nature Neurosci. paper and Fig. $6 \mathrm{a}$ of the J. Neurosci. paper).

We greatly regret that neither of these papers cites the other, and that we failed to inform the editors of either Nature Neuroscience or Journal of Neuroscience of the existence of another closely related paper that was under consideration elsewhere. We also regret that an important result presented in the J. Neurosci. paper (the effect of a second lesion rostral to the first) was mistakenly described in the Nature Neurosci. paper as "W.J.Z., in preparation" while it was already in press at Journal of Neuroscience.

We apologize to the editors, referees and readers of both Nature Neuroscience and Journal of Neuroscience for these errors, and for any confusion which we may have caused.

A similar correction has been submitted to Journal of Neuroscience.

Martin E. Schwab, Michaela Thallmair, Werner Z'Graggen and Gerlinde Metz. 


\section{Visual search for motion-in-depth: stereomotion does not 'pop out' from disparity noise}

Julie M. Harris ${ }^{1}$, Suzanne P. McKee ${ }^{2}$ and Scott N. J. Watamaniuk ${ }^{3}$

Nature Neurosci. 1, 165-168 (1998).

Because of an editorial error, the affiliation given for JMH was incomplete. The correct affiliations are:

${ }^{1}$ Address until 30 June 1998: Department of Pharmacology and Centre for Neuroscience, University of Edinburgh, Crichton St., Edinburgh, EH8 9LE, UK
Address after 1 July 1998: Department of Psychology, Ridley Building, Claremont Place, University of Newcastle, Newcastle upon Tyne, NE1 7RU, UK

\section{Visual features that vary together over time group together over space}

David Alais, Randolph Blake and Sang-Hun Lee

Nature Neurosci. 1, 160-164 (1998).

The following paragraphs were inadvertently omitted from the results section. The end of that section should read:

(page 162)...More importantly, correlated contrast modulation still produced greater coherence compared to the uncorrelated case even when the two gratings differed in contrast.

Contrast modulation of a drifting grating introduces additional temporal frequencies in the stimulus, besides those associated with the smooth drift rate. Because the contrast modulations were purposefully random, the fluctuations in temporal frequency were also random. In the case of our two-component plaid, random contrast modulations effectively modulate the length (but not the direction) of the component vectors, and these random fluctuations in vector length could be perceived as irregularities in speed, or motion 'jerkiness.' For uncorrelated contrast modulations, the changes in vector length would differ for the two components and, therefore, possibly complicate the grouping process. But why would irregular, jerky motion that is correlated among components increase perceived coherence, the result we obtained? One might argue that correlated jerkiness leads the visual system intelligently to deduce that the jerky components belong to the same object.

To test this possibility, we created a plaid consisting of two components that did not modulate in contrast. In two conditions, we explicitly selected a new drift-rate every $40 \mathrm{~ms}$ from a set of seven ranging from $4-8 \mathrm{~Hz}$; this caused motion to appear jerky. In one of these conditions, the random fluctuations in drift-rate were correlated, and in the other they were uncorrelated. In a third condition, the component gratings drifted smoothly at a constant rate of $6 \mathrm{~Hz}$ throughout the observation period. Observers again tracked periods of global coherence during 60-second observation periods. Jerky motion had no effect on perceived coherence, which was equivalent for all three conditions. Accordingly, we conclude that jerkiness is not responsible for the increased coherence produced by correlated contrast modulations.

\section{Cocaine self-administration in dopamine-transporter knockout mice}

Beatriz A. Rocha, Fabio Fumagalli, Raul R. Gainetdinov, Sara R. Jones, Robert Ator, Bruno Giros, Gary W. Miller and Marc G. Caron

Nature Neurosci. 1, 132-137 (1998).

On page 134, Fig. 4 was inadvertently printed in black and white instead of color. The corrected version is printed below.
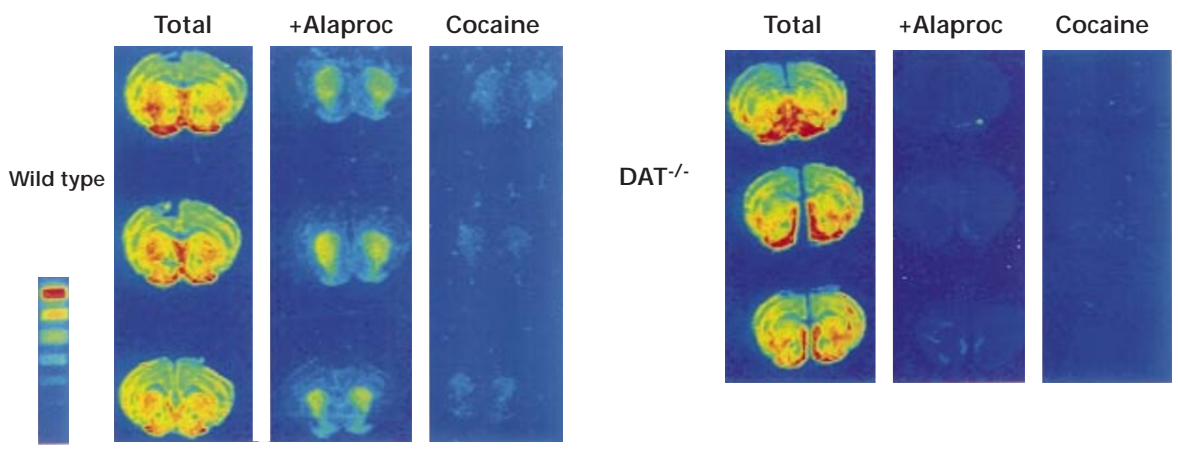


\section{Presynaptic modulation of CA3 network activity}

Kevin J. Staley, Mark Longacher, Jaideep S. Bains and Audrey Yee

Nature Neurosci. 1, 201-209 (1998).

Because of a printing error, Figs $4 c, 5 c$ and 6 were not properly reproduced. The corrected versions are printed below.

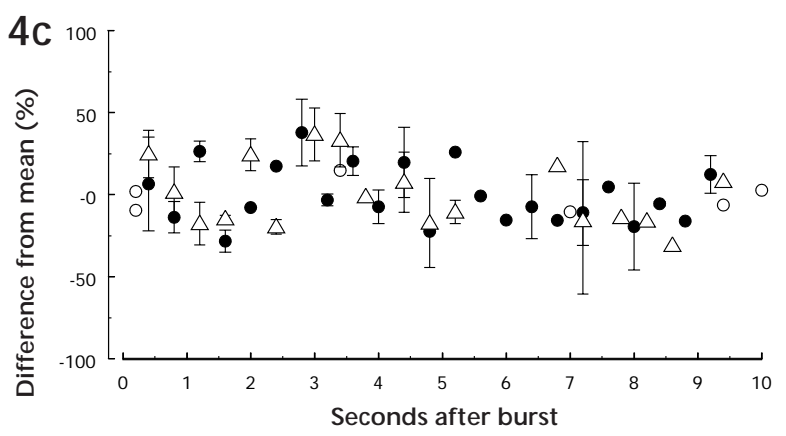

6a

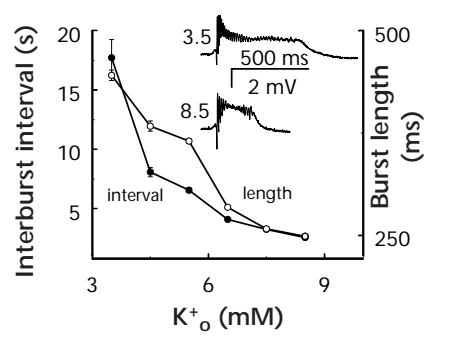

C
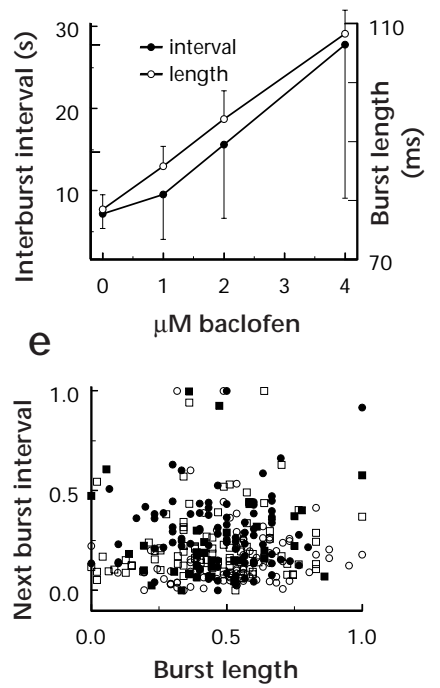

b

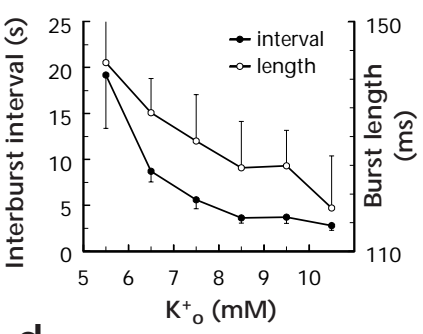

d
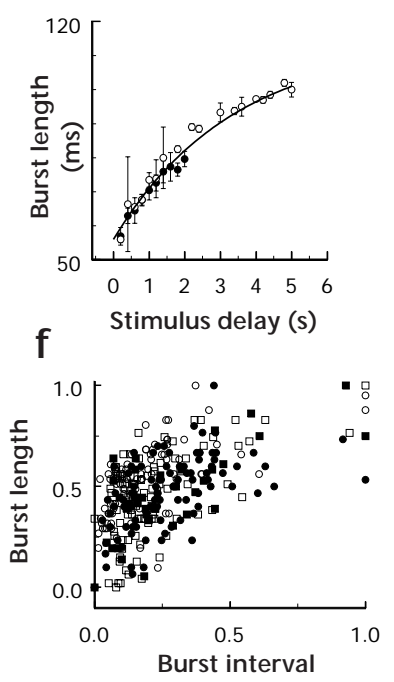

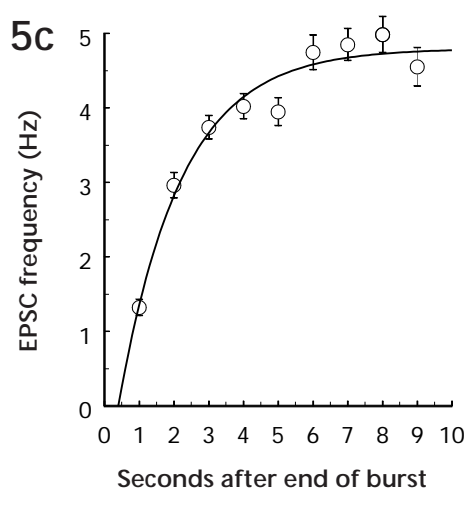

In addition, the penultimate sentence in the legend to Fig. $4 \mathrm{~b}$ was inadvertently omitted. The corrected legend should read:

Fig. 4..... (b) The experiment shown in (a) was repeated in a slice in which CA3 bursting was induced by tetanic stimulation of the CA3 pyramidal cell layer. The average interburst interval in this preparation was 13 seconds, which permitted longer delays between the end of a burst and glutamate application. As in (a), glutamate application at delays closer to the interburst interval triggered a burst more rapidly, but the initial response to glutamate was unchanged (inset). In this cell, bursts triggered calcium escape spikes. Glutamate was applied after every fourth burst in (b) and (c). 